Ann. Inst. Statist. Math.

Vol. 45, No. 4, 655-664 (1993)

\title{
A MEASURE OF ROTATABILITY FOR SECOND ORDER RESPONSE SURFACE DESIGNS
}

\author{
SUNG H. PARK ${ }^{1}$, JUN H. LIM ${ }^{1}$ AND YASUMASA BABA ${ }^{2}$ \\ ${ }^{1}$ Department of Computer Science and Statistics, Seoul National University, \\ San 56-1, Shinrim-Dong, Kwanak-ku, Seoul 151-742, Korea \\ ${ }^{2}$ The Institute of Statistical Mathematics, 4-6-7 Minami-Azabu, Minato-ku, Tokyo 106, Japan
}

(Received March 28, 1991; revised November 16, 1992)

\begin{abstract}
The concept of rotatability introduced by Box and Hunter (1957, Ann. Math. Statist., 28, 195-241) is an important design criterion for response surface design. Recently, a few measures of rotatability that enable us to assess the degree of rotatability for a given response surface design have been introduced. In this paper, a new measure of rotatability for second order response surface designs is suggested, and illustrated for $3^{k}$ factorial design and central composite design. Also a short comparison is made between the proposed measure with the previously suggested measures.
\end{abstract}

Key words and phrases: Rotatability, measure, response surface design, factorial design, central composite design.

\section{Introduction}

Suppose that an experimenter is concerned with a system involving some response $\eta$ which depends on several independent variables $\xi_{1}, \ldots, \xi_{k}$. In general, the functional relationship between the independent variables and the mean response can be written as $\eta=f\left(\xi_{1}, \ldots, \xi_{k}\right)$, where the explicit form of $f$ is unknown or expremely complicated. Response Surface Methodology (RSM) often involves the approximation of $f$ by a low order polynomial in some region of the independent variables. Usually, the original variables $(\xi ' s)$ are coded to the design variables $(x$ 's) in order to locate the origin to the center of region, the latter normally being simple linear functions of the former.

In this paper, we will concentrate on the second order model

$$
\eta(\boldsymbol{x})=\beta_{0}+\sum_{i=1}^{k} \beta_{i} x_{i}+\sum_{i=1}^{k} \beta_{i i} x_{i}^{2}+\sum_{i<j}^{k} \beta_{i j} x_{i} x_{j}
$$

which may be written in matrix notation as

$$
\eta(x)=x_{s}^{\prime} \beta
$$


in which

$$
\begin{aligned}
& x^{\prime}=\left(x_{1}, x_{2}, \ldots, x_{k}\right), \\
& x_{s}^{\prime}=\left(1, x_{1}, x_{2}, \ldots, x_{k}, x_{1}^{2}, x_{2}^{2}, \ldots, x_{k}^{2}, x_{1} x_{2}, \ldots, x_{k-1} x_{k}\right)
\end{aligned}
$$

and $\beta$ is the $m \times 1$ column vector corresponding to the coefficients, where $m=$ $(k+1)(k+2) / 2$.

The coefficients in the polynomial are to be estimated, by the method of least squares, from $N$ observations on the response variable,

$$
y_{u}=\eta\left(\boldsymbol{x}_{u}\right)+\epsilon_{u}, \quad u=1,2, \ldots, N
$$

where $\epsilon_{u}$ 's are assumed to be uncorrelated and have zero means and constant variance, $\sigma^{2}$. The $\beta$ is then estimated by the method of least squares as follows

$$
b=\left(X^{\prime} X\right)^{-1} X^{\prime} y
$$

in which $X$ is the $N \times m$ matrix of values of the $m$ elements of $\boldsymbol{x}_{s}$ 's taken at the design points and $y$ is the $N \times 1$ matrix of observations.

The predicted response value at a particular point $\boldsymbol{x}^{\prime}=\left(x_{1}, x_{2}, \ldots, x_{k}\right)$ in the region of interest $R$ will be denoted by $\hat{y}(\boldsymbol{x})$. This value is obtained by substituting the elements of $\beta$ in the model (1.1) by the corresponding elements of $b$ in (1.2), i.e., $\hat{y}(\boldsymbol{x})=\boldsymbol{x}_{s}^{\prime} \boldsymbol{b}$. The variance function of the predicted value $\hat{y}$ at any point $\boldsymbol{x}^{\prime}=\left(x_{1}, x_{2}, \ldots, x_{k}\right)$ is well known as

$$
\operatorname{Var}(\hat{y}(\boldsymbol{x}))=\boldsymbol{x}_{s}^{\prime}\left(X^{\prime} X\right)^{-1} \boldsymbol{x}_{s} \sigma^{2}
$$

$\operatorname{Var}(\hat{y}(\boldsymbol{x}))$ thus depends on the particular values of the independent variables through the vector $x_{s}^{\prime}$, and also depends on the design through the matrix $\left(X^{\prime} X\right)^{-1}$.

A design $D$ is said to be rotatable if the variance function (1.3) is a function of only $r^{2}=x_{1}^{2}+x_{2}^{2}+\cdots+x_{k}^{2}$, i.e., the variance of $\hat{y}$ is a function of only distance of $\boldsymbol{x}$ from the origin, not of direction. Thus, when a design is rotatable, the prediction variance is the same at all points $\boldsymbol{x}$ that are equidistant from the design center. Consequently, in the space of the input variables, surfaces of constant prediction variance from concentric hyperspheres.

A work which has generated initial interest in the use of RSM is a paper by Box and Wilson (1951). In recent years, interests in RSM have been increased and books on this subject have been written by some authors such as Myers (1976), Box and Draper (1987) and Khuri and Cornell (1987).

The concepts of rotatability was first introduced by Box and Hunter (1957). Since it was first introduced, it has become an important design criterion. If the circumstances are such that exact rotatability is unattainable, it is still a good idea to make the design nearly rotatable. Thus it is important to know if a particular design is rotatable or, if it is not, to know how rotatable it is. Analogous to rotatability, the concept of slope-rotatability has been advanced by Hader and Park 
(1978) and Park (1987). In this concept, the variance of $\partial \hat{y}(\boldsymbol{x}) / \partial x_{i}(i=1, \ldots, k)$ are equal for all $x$ equidistant from the design origin.

Recently, Khuri (1988), Draper and Guttman (1988), and Draper and Pukelsheim (1990) suggest the measures of rotatability that enable us to assess the degree of rotatability for a given design. In this paper, another measure of rotatability, $R_{k}(D)$, is introduced and illustrated.

\section{Proposed measure of rotatability}

In this chapter, a general measure of rotatability is proposed. This measure will enable us to appreciate the degree of rotatability for a given response surface design. Let

$$
V(\boldsymbol{x})=\frac{N}{\sigma^{2}} \operatorname{Var}(\hat{y}(\boldsymbol{x}))
$$

where $\operatorname{Var}(\hat{y}(\boldsymbol{x}))=\boldsymbol{x}_{s}^{\prime}\left(X^{\prime} X\right)^{-1} \boldsymbol{x}_{s} \sigma^{2}$.

In the $k$-dimensional space $(k \geq 2), V(\boldsymbol{x})$ can be expressed in terms of spherical coordinates of $\left(\rho, \phi_{1}, \phi_{2}, \ldots, \phi_{k-2}, \theta\right)$ where

$$
\begin{aligned}
& x_{1}=\rho \cos \phi_{1}, \\
& x_{2}=\rho \sin \phi_{1} \cos \phi_{2},
\end{aligned}
$$

$$
\begin{aligned}
x_{k-1} & =\rho \sin \phi_{1} \sin \phi_{2} \cdots \sin \phi_{k-2} \cos \theta, \\
x_{k} & =\rho \sin \phi_{1} \sin \phi_{2} \cdots \sin \phi_{k-2} \sin \theta
\end{aligned}
$$

and $\rho \geq 0,0 \leq \phi_{1}, \phi_{2}, \ldots, \phi_{k-2} \leq \pi, 0 \leq \theta<2 \pi$. (See Fleming (1977), p. 218.) The absolute value of the Jacobian of this transformation is

$$
|J|=\rho^{k-1} \sin ^{k-2} \phi_{1} \sin ^{k-3} \phi_{2} \cdots \sin ^{2} \phi_{k-3} \sin \phi_{k-2} .
$$

If we substitute (2.2) into (2.1), then (2.1) will be expressed as a function of $\rho, \phi_{1}, \phi_{2}, \ldots, \phi_{k-2}, \theta$, i.e., $V(\boldsymbol{x})=w\left(\rho, \phi_{1}, \phi_{2}, \ldots, \phi_{k-2}, \theta\right)$. Let

$$
\bar{w}(\rho)=\frac{1}{I_{k}} \int_{0}^{2 \pi} \int_{0}^{\pi} \ldots \int_{0}^{\pi} w\left(\rho, \phi_{1}, \phi_{2}, \ldots, \phi_{k-2}, \theta\right) d \Omega
$$

where $d \Omega=\sin ^{k-2} \phi_{1} \cdots \sin \phi_{k-2} d \phi_{1} d \phi_{2} \cdots d \phi_{k-2} d \theta$, and

$$
I_{k}=\int_{0}^{2 \pi} \int_{0}^{\pi} \cdots \int_{0}^{\pi} d \Omega=\frac{2 \pi^{k / 2}}{\Gamma(k / 2)}
$$

$\bar{w}(\rho)$ means the averaged value of $V(x)$ over all the points on the hypersphere of radius $\rho$ centered at the origin. To be rotatable,

$$
w\left(\rho, \phi_{1}, \phi_{2}, \ldots, \phi_{k-2}, \theta\right)=\bar{w}(\rho) \quad \text { for all } \rho, \phi_{i}, \theta
$$


For a given design, the discrepancy from rotatability at $\rho$ can be expressed as

$$
h(\rho)=\int_{0}^{2 \pi} \int_{0}^{\pi} \cdots \int_{0}^{\pi}\left[w\left(\rho, \phi_{1}, \phi_{2}, \ldots, \phi_{k-2}, \theta\right)-\bar{w}(\rho)\right]^{2} d \Omega .
$$

If the region of interest is $0 \leq \rho \leq 1$, the proposed measure will be

$$
P_{k}(D)=\frac{1}{1+R_{k}(D)}
$$

where

$$
R_{k}(D)=\frac{1}{E_{k}} \int_{0}^{1} \rho^{k-1} h(\rho) d \rho,
$$

and $E_{k}$ is a positive constant depending only on $k$. Let us take $E_{k}$ to be

$$
E_{k}=\int_{0}^{1} \rho^{k-1} I_{k} d \rho=\frac{1}{k} I_{k}
$$

for convinience. By this way, $R_{k}(D)$ represents the average of $(w-\bar{w})^{2}$ over the region of integration. $P_{k}(D)$ is 1 if and only if a design is rotatable, and it is smaller than one for a nonrotatable design. Note that $P_{k}(D)$ is invariant with respect to the rotation of the coordinate axes, since $\bar{w}(\rho), h(\rho)$ and $R_{k}(D)$ are invariant with respect to the rotation of the coordinate axes.

Now, we introduce the following fact which is useful for evaluating our measure.

(1) $\int d \Omega=2 \pi^{k / 2} / \Gamma(k / 2)$,

(2) $\int x_{i}^{2} d \Omega=\rho^{2} I_{k} / k$

(3) $\int x_{i}^{2} x_{j}^{2} d \Omega=(1 / 3) \int x_{i}^{4} d \Omega=\rho^{4} I_{k} / k(k+2)(i \neq j)$,

(4) $\int x_{i}^{2} x_{j}^{2} x_{l}^{2} d \Omega=(1 / 3) \int x_{i}^{4} x_{j}^{2} d \Omega=(1 / 15) \int x_{i}^{6} d \Omega=\rho^{6} I_{k} / k(k+2)(k+4)$ $(i \neq j \neq l)$,

(5) $\int x_{i}^{2} x_{j}^{2} x_{l}^{2} x_{m}^{2} d \Omega=(1 / 3) \int x_{i}^{4} x_{j}^{2} x_{l}^{2} d \Omega=(1 / 9) \int x_{i}^{4} x_{j}^{4} d \Omega=(1 / 15) \int x_{i}^{6} x_{j}^{2} d \Omega$ $=(1 / 105) \int x_{i}^{8} d \Omega=\rho^{8} I_{k} / k(k+2)(k+4)(k+6)(i \neq j \neq l \neq m)$

where $i, j, l, m$ could be $1,2, \ldots, k$, and $\int$ means $\int_{0}^{2 \pi} \int_{0}^{\pi} \cdots \int_{0}^{\pi}$. The values of other integrals where at least one $x_{i}$ has an odd exponent are all zeros.

In comparing designs, we have to consider the scaling of designs. The traditional way of scaling is to set $1 / N \sum_{u=1}^{N} x_{i u}^{2}=1$. This was used by Box and Hunter (1957), and Khuri (1988). In this paper, as was adapted by Draper and Pukelsheim (1990), we scale designs so that all of the points lie inside or on the unit sphere. Therefore, if we have a set of points

$$
\boldsymbol{x}_{i}^{\prime}=\left(x_{1 i}, x_{2 i}, \ldots, x_{k i}\right) \quad i=1,2, \ldots, N,
$$

then the scaled point, $g x_{i}$, should satisfy

$$
0 \leq g \sqrt{\left(x_{1 i}\right)^{2}+\left(x_{2 i}\right)^{2}+\cdots+\left(x_{k i}\right)^{2}} \leq 1 \quad i=1,2, \ldots, N .
$$

One advantage of this is that, when we add center points, the remaining points do not have to be rescaled. For the $3^{2}$-factorial design, the scaling factor should be $g=1 / \sqrt{2} \doteq 0.7071$. 
3. Illustration of the proposed measure

\section{$3.13^{k}$ factorial design}

For the $3^{k}$ factorial design without replications, it can be shown that

$$
\begin{aligned}
& v_{0}=\frac{2 k+1}{3^{k}} \sigma^{2}, \\
& v_{i}=\frac{1}{2 \times 3^{k-1}} \sigma^{2} \quad(i=1,2, \ldots, k), \\
& v_{i i}=\frac{1}{2 \times 3^{k-2}} \sigma^{2} \quad(i=1,2, \ldots, k), \\
& v_{i j}=\frac{1}{4 \times 3^{k-2}} \sigma^{2} \quad(i \neq j), \\
& c_{0, i i}=-\frac{1}{3^{k-1}} \sigma^{2} \quad(i=1,2, \ldots, k)
\end{aligned}
$$

where $v_{0}, v_{i}, v_{i i}, v_{i j}$ and $c_{0, i i}$ denote $\operatorname{Var}\left(b_{0}\right), \operatorname{Var}\left(b_{i}\right), \operatorname{Var}\left(b_{i i}\right), \operatorname{Var}\left(b_{i j}\right)$ and $\operatorname{Cov}\left(b_{0}, b_{i i}\right)$, respectively, and $\boldsymbol{b}^{\prime}=\left(b_{0}, b_{1}, \ldots, b_{k}, b_{11}, \ldots, b_{k k}, b_{12}, \ldots, b_{k-1, k}\right)$ which is explained in (1.2).

The other values of variances and covariances not mentioned above are all zeros. Thus, we can obtain the following results,

$$
\begin{aligned}
V(\boldsymbol{x})= & \frac{N}{\sigma^{2}} \operatorname{Var}(\hat{y}(\boldsymbol{x})) \\
= & \frac{N}{\sigma^{2}}\left[v_{0}+\left(v_{1}+2 c_{0,11}\right) \rho^{2}+v_{11} \rho^{4}\right. \\
& \left.\quad+\left(v_{12}-2 v_{11}\right)\left(x_{1}^{2} x_{2}^{2}+\cdots+x_{k-1}^{2} x_{k}^{2}\right)\right]
\end{aligned}
$$

and,

$$
\begin{aligned}
\bar{w}(\rho) & =\frac{1}{I_{k}} \int V(\boldsymbol{x}) d \Omega \\
& =\frac{N}{\sigma^{2}}\left[v_{0}+\left(v_{1}+2 c_{0,11}\right) \rho^{2}+v_{11} \rho^{4}+\left(v_{12}-2 v_{11}\right)\left(\begin{array}{l}
k \\
2
\end{array}\right) \frac{\rho^{4}}{k(k+2)}\right] .
\end{aligned}
$$

Therefore,

$$
\begin{aligned}
& {\left[w\left(\rho, \phi_{1}, \phi_{2}, \ldots, \phi_{k-2}, \theta\right)-\bar{w}(\rho)\right]^{2}} \\
& =\left(\frac{N}{\sigma^{2}}\right)^{2}\left(v_{12}-2 v_{11}\right)^{2}\left[\left(x_{1}^{2} x_{2}^{2}+\cdots+x_{k-1}^{2} x_{k}^{2}\right)^{2}+\frac{(k-1)^{2}}{4(k+2)^{2}} \rho^{8}\right. \\
& \left.\quad-\frac{(k-1)}{(k+2)}\left(x_{1}^{2} x_{2}^{2}+\cdots+x_{k-1}^{2} x_{k}^{2}\right) \rho^{4}\right] .
\end{aligned}
$$

From this, for (2.4) we can obtain

$$
h(\rho)=\left(\frac{N}{\sigma^{2}}\right)^{2} \frac{6\left(v_{12}-2 v_{11}\right)^{2}(k-1) \rho^{8} I_{k}}{(k+2)^{2}(k+4)(k+6)} .
$$


Thus, from (2.6)

$$
R_{k}(D)=\frac{k}{I_{k}}\left(\frac{N}{\sigma^{2}}\right)^{2} \frac{6\left(v_{12}-2 v_{11}\right)^{2}(k-1) I_{k}}{(k+2)^{2}(k+4)(k+6)(k+8)} .
$$

If we take the scaling factor $g=1 / \sqrt{k}$ in the $3^{k}$-factorial design, the variances $v_{12}$ and $v_{11}$ become $\left(1 / g^{4}\right) v_{12}$ and $\left(1 / g^{4}\right) v_{11}$, respectively, after scaling. So

$$
R_{k}(D)=\left(\frac{N}{\sigma^{2}}\right)^{2} \frac{6 k\left(v_{12}-2 v_{11}\right)^{2}(k-1)}{(k+2)^{2}(k+4)(k+6)(k+8) g^{8}} .
$$

Table 1 gives the values of $P_{k}(D)=1 /\left[1+R_{k}(D)\right]$ for various $k$, which indicates that as $k$ increases $3^{k}$-factorial designs deviate more from rotatability.

Table 1 . Values of $P_{k}(D)$ for $3^{k}$-factorial designs.

\begin{tabular}{cccccc}
\hline$k$ & 2 & 3 & 4 & 5 & 6 \\
\hline$P_{k}(D)$ & 0.468 & 0.115 & 0.040 & 0.018 & 0.010 \\
\hline
\end{tabular}

\subsection{Central composition designs}

In general, the Central Composition Design (CCD) consists of $2^{k}$ or a fraction of $2^{k}$ factorial points $( \pm 1, \pm 1, \ldots, \pm 1), 2 k$ axial points of the form $( \pm \alpha, 0, \ldots, 0)$, etc., and a center point $(0,0, \ldots, 0)$. The center point may be replicated $n_{0}$ times. Thus the total number $N$ of experimental points can be written as

$$
N=F+2 k+n_{0}=F+T
$$

where $F$ is the number of factorial points $\left(F=2^{k}\right.$ if a full factorial is used and $F=2^{k-p}(p \geq 1)$ if a fractional factorial is used), and $T=2 k+n_{0}$. For the CCD, it can be shown that for any fractional factorial configurations,

$$
\begin{aligned}
& v_{0}=\frac{k F+2 \alpha^{4}}{2 \alpha^{4}(F+T-2 k)-4 \alpha^{2} k F+k F T} \sigma^{2}, \\
& v_{i}=\frac{1}{F+2 \alpha^{2}} \sigma^{2} \quad(i=1,2, \ldots, k), \\
& v_{i j}=\frac{1}{F} \sigma^{2} \quad(i \neq j), \\
& v_{i i}=\frac{\left\{2 \alpha^{4}+4(1-k) \alpha^{2}+(k-1) T\right\} F+2 \alpha^{4}(T-2 k+2)}{2 \alpha^{4}\left\{\left(2 \alpha^{4}+k T-4 k \alpha^{2}\right) F+2 \alpha^{4}(T-2 k)\right\}} \sigma^{2} \\
& c_{i i, j j}=\frac{4 \alpha^{4}+4 \alpha^{2} F-T F \quad(i=1,2, \ldots, k),}{2 \alpha^{4}\left\{\left(2 \alpha^{4}+k T-4 k \alpha^{2}\right) F+2 \alpha^{4}(T-2 k)\right\}} \sigma^{2} \quad(i \neq j), \\
& c_{0, i i}=\frac{-\left(F+2 \alpha^{2}\right)}{2 \alpha^{4}(F+T-2 k)-4 \alpha^{2} k F+k F T} \sigma^{2} \quad(i=1,2, \ldots, k)
\end{aligned}
$$


where $c_{i i, j j}=\operatorname{Cov}\left(b_{i i}, b_{j j}\right)$ and $c_{0, i i}=\operatorname{Cov}\left(b_{0}, b_{i i}\right)$. Thus, we can obtain the following results:

$$
\begin{aligned}
V(x)=\frac{N}{\sigma^{2}}[ & v_{0}+\left(v_{1}+2 c_{0,11}\right) \rho^{2}+v_{11} \rho^{4} \\
& \left.\quad+\left(v_{12}+2 c_{11,22}-2 v_{11}\right)\left(x_{1}^{2} x_{2}^{2}+\cdots+x_{k-1}^{2} x_{k}^{2}\right)\right] \\
\bar{w}(\rho)=\frac{N}{\sigma^{2}} & {\left[v_{0}+\left(v_{1}+2 c_{0,11}\right) \rho^{2}+v_{11} \rho^{4}\right.} \\
& \left.+\left(v_{12}+2 c_{11,22}-2 v_{11}\right) \frac{(k-1)}{2(k+2)} \rho^{4}\right]
\end{aligned}
$$

and

$$
h(\rho)=\left(\frac{N}{\sigma^{2}}\right)^{2} \frac{6\left(v_{12}+2 c_{11,22}-2 v_{11}\right)^{2}(k-1) I_{k} \rho^{8}}{(k+2)^{2}(k+4)(k+6)} .
$$

In the CCDs, the scaling factor, $g$, should be

$$
g= \begin{cases}1 / \alpha, & \text { if } \alpha \geq \sqrt{k} \\ 1 / \sqrt{k}, & \text { if } \alpha<k\end{cases}
$$

Then

$$
R_{k}(D)=\left(\frac{N}{\sigma^{2}}\right)^{2} \frac{6 k\left(v_{12}+2 c_{11,22}-2 v_{11}\right)^{2}(k-1)}{(k+2)^{2}(k+4)(k+6)(k+8) g^{8}}
$$

Table 2 gives the values of $P_{k}(D)$ for various $k, p$ and $\alpha$.

Figures 1 through 3 give the contour plot ov $V(\boldsymbol{x})$ for the CCDs $(k=2, p=0$, $n_{0}=1$ ) with $\alpha=1.00,2.00$ and 3.00. Note that when $\alpha=1.00$, the CCD is the $3^{2}$ factorial design.

Table 2. Values of $P_{k}(D)$ for CCDs for various $k, p$ and $\alpha\left(n_{0}=1\right)$.

\begin{tabular}{cccccccc}
\hline \multirow{2}{*}{$\alpha$} & $k=2$ & $k=3$ & $k=4$ & $k=5$ & $k=5$ & $k=6$ & $k=6$ \\
& $p=0$ & $p=0$ & $p=0$ & $p=0$ & $p=1$ & $p=1$ & $p=2$ \\
\hline 1.00 & 0.4675 & 0.0333 & 0.0034 & 0.0005 & 0.0013 & 0.0002 & 0.0006 \\
1.50 & 0.9911 & 0.8339 & 0.1413 & 0.0162 & 0.0595 & 0.0082 & 0.0292 \\
2.00 & 0.4675 & 0.6814 & 1.0000 & 0.3177 & 1.0000 & 0.1890 & 1.0000 \\
2.50 & 0.0932 & 0.1242 & 0.2699 & 0.8537 & 0.2576 & 0.8583 & 0.2548 \\
3.00 & 0.0209 & 0.0250 & 0.0445 & 0.1081 & 0.0419 & 0.1117 & 0.0413 \\
3.50 & 0.0059 & 0.0067 & 0.0108 & 0.0205 & 0.0102 & 0.0212 & 0.0100 \\
Rotatable $\alpha$ & 1.41 & 1.68 & 2.00 & 2.38 & 2.00 & 2.38 & 2.00 \\
\hline
\end{tabular}




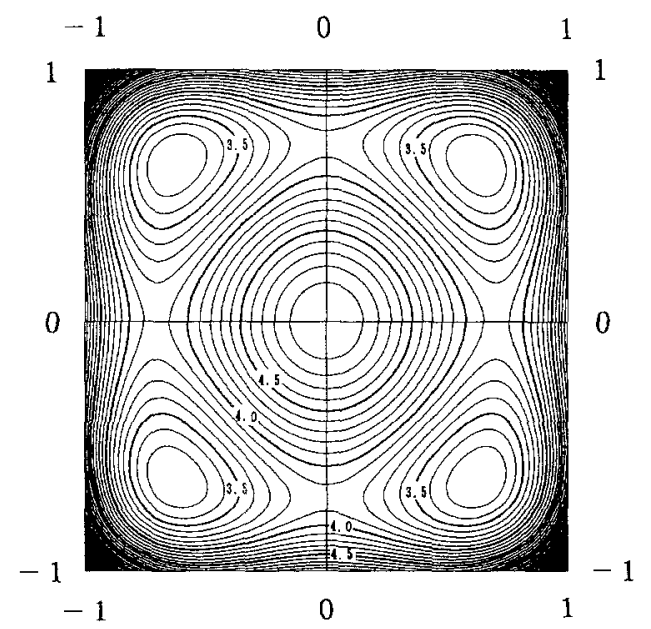

Fig. 1. Contour plot of $V(x)$ for $\operatorname{CCD}\left(k=2, p=0, n_{0}=1, \alpha=1.00\right)$.

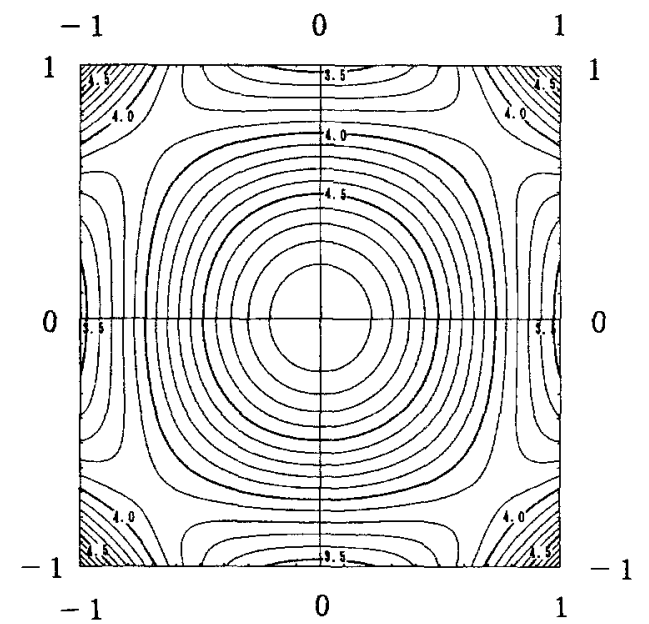

Fig. 2. Contour plot of $V(x)$ for $\operatorname{CCD}\left(k=2, p=0, n_{0}=1, \alpha=2.00\right)$.

\section{Comparison of rotatability measures}

It is of interest to compare the proposed measure $P_{k}(D)$ with the measures of Khuri (1988), Draper and Guttman (1988) and Draper and Pukelsheim (1990). Both Khuri's measure and Draper and Pukelsheim's measure compare the form of $X^{\prime} X$ matrix from regression model with that of rotatable design, and can be used with any model of order $d(\geq 1)$. However, both measures do not provide information about variance contour shape. On the other hand, Draper and Guttman provide an index that characterizes the general overall shape of variance contours. But it is difficult to apply this index to general nonsymmetric designs.

For the usefulness of our proposed measure, we want to mention the following facts. 


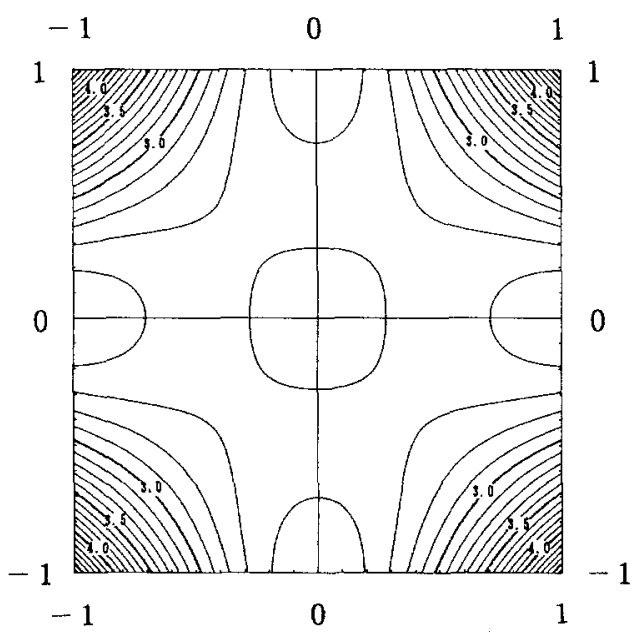

Fig. 3. Contour plot of $V(\boldsymbol{x})$ for $\operatorname{CCD}\left(k=2, p=0, n_{0}=1, \alpha=3.00\right)$.

Table 3. Comparison of the four measures of rotatability.

\begin{tabular}{lcccc}
\hline \multicolumn{1}{c}{ Criteria } & Khuri & $\begin{array}{c}\text { Draper \& } \\
\text { Guttman }\end{array}$ & $\begin{array}{c}\text { Draper \& } \\
\text { Pukelsheim }\end{array}$ & $P_{k}(D)$ \\
\hline $\begin{array}{l}\text { Applicability } \\
\text { to asymmetric } \\
\text { design }\end{array}$ & Yes & No & Yes & Yes \\
$\begin{array}{l}\text { Invariance } \\
\begin{array}{l}\text { w.r.t the } \\
\text { design rotation }\end{array}\end{array}$ & No & No & Yes & Yes \\
$\begin{array}{l}\text { Information } \\
\text { about variance } \\
\text { contour shape }\end{array}$ & No & Yes & No & No \\
$\begin{array}{l}\text { Range } \\
\begin{array}{l}\text { Order of the } \\
\text { model to which the } \\
\text { measure applies }\end{array}\end{array}$ & $0 \sim 100$ & $0 \sim+\infty$ & $0 \sim 1$ & $0 \sim 1$ \\
\hline
\end{tabular}

First of all, we can easily obtain the variance contour $V(x)$ like Figs. 1-3 from the equations (3.1) and (3.6), since $V(\boldsymbol{x})$ is expressed in terms of spherical coordinates. Next, it is true that the proposed measure is only applied to second order designs. However, rotatability is mainly discussed for second order response surface designs, and this limitation does not pose any practical problems for the usefulness of this measure in response surface methodology. Last of all, we have a computer program, named SROTA, which computes $P_{k}(D)$ for any second-order design. This program can be obtained from the authors upon request. For a given 
design, this program obtains $V(\boldsymbol{x})$, compute $\bar{w}(\rho)$ and $h(\rho)$, and then calculate $R_{k}(D)$.

In Table 3, we make a comparison of the four measures of rotatability on the basis of five criteria.

\section{Acknowledgements}

We are very much indebted to the editor and referees who contributed valuable suggestions and comments that helped improve this paper very much.

\section{REFERENCES}

Box, G. E. P. and Draper, N. R. (1987). Empirical Model-Building and Response Surface, Wiley, New York.

Box, G. E. P. and Hunter, J. S. (1957). Multifactor experimental designs for exploring response surfaces, Ann. Math. Statist., 28, 195-241.

Box, G. E. P. and Wilson, K. B. (1951.). On the experimental attainment of optimum conditions, J. Roy. Statist. Soc. Ser. B, 13, 1-45.

Draper, N. R. and Guttman, I. (1988). An index of rotatability, Technometrics, 30, 105-111.

Draper, N. R. and Pukelsheim, F. (1990). Another look at rotatability, Technometrics, 32, 195-202.

Fleming, W. (1977). Functions of Several Variables, 2nd ed., Springer, New York.

Hader, R. J. and Park, S. H. (1978). Slope-rotatable central composite designs, Technometrics, 20, 413-417.

Khuri, A. I. (1988). A measure of rotatability for response surface designs, Technometrics, 30, $95-104$.

Khuri, A. I. and Cornell, J. A. (1987). Response Surfaces: Designs and Analyses, Marcel Dekker, New York.

Myers, R. H. (1976). Response Surface Methodology, Edwards Brothers, Ann Arbor, Michigan.

Park, S. H. (1987). A class of multifactor designs for estimating the slope of response surfaces, Technometrics, 29, 449-453. 